\title{
Influencia del estrés por déficit hídrico sobre el rendimiento de cultivo de trigo (Triticum aestivum) bajo
} condiciones controladas en Chachapoyas, Amazonas

\section{Influence of stress due to water deficit on the yield of wheat crop (Triticum aestivum) under controlled conditions in Chachapoyas, Amazonas}

\author{
Juan C. Neri ${ }^{1}$ (I) , Eyner Huaman Huaman ${ }^{1}\left(\mathbb{D}\right.$, Roicer Collazos Silva ${ }^{1} \mathbb{D}$, Manuel Oliva ${ }^{1} \mathbb{D}$, Manuel Alejandro Ix-Balam ${ }^{2}$
}

\section{RESUMEN}

El presente estudio tuvo como objetivo evaluar el efecto del estrés por déficit hídrico sobre características morfológicas (altura de planta, longitud de hoja y longitud de raíz) y agronómicas (número de granos/espiga, número de espigas $/ \mathrm{m}^{2}$ y rendimiento) de trigo (Triticum aestivum). Se evaluaron cuatro regímenes de riego $(25 \%, 50 \%, 75 \%$ y $100 \%$ ) y dos variedades de trigo (Gavilán y San Isidro), para lo cual se utilizó un diseño experimental completamente aleatorizado con arreglo factorial. La siembra se realizó en macetas con sustrato a base de tierra agrícola y humus (3:1), luego de 30 días se aplicaron los regímenes de riego establecidos en base a la ETc y requerimiento de riego del cultivo. Los datos se sometieron al análisis de varianza y la prueba de Duncan $(\mathrm{p} \leq 0,05)$. Los resultados mostraron diferencias significativas entre tratamientos. La aplicación de riego al 75 y $100 \%$ del requerimiento permitió alcanzar las mayores alturas de planta con promedios entre 53,37 a $57,44 \mathrm{~cm}$ y longitud de hoja con valores entre 11,89 y $11,37 \mathrm{~cm}$. Además, estos tratamientos han mostrado mejor comportamiento agronómico, reportando entre 2500 a 3500 granos $/ \mathrm{m}^{2} \mathrm{y}$, por lo tanto, mejores rendimientos $\left(>120 \mathrm{~g} / \mathrm{m}^{2}\right)$.

Palabras claves: estrés hídrico, déficit hídrico, evapotranspiración, rendimiento, Triticum aestivum

\begin{abstract}
The present study aimed to evaluate the effect of stress due to water deficit on morphological (plant height, leaf length and root length) and agronomic (number of grains/spike, number of spikes $/ \mathrm{m}^{2}$ and yield) characteristics of wheat (Triticum aestivum). Four irrigation regimes $(25 \%, 50 \%, 75 \%$ and $100 \%)$ and two varieties of wheat (Gavilán and San Isidro) were evaluated, for which a completely randomized experimental design with factorial arrangement was used. The sowing was carried out in pots with substrate based on agricultural land and humus (3:1), after 30 days the irrigation regimes established based on the ETc and irrigation requirement of the crop were applied. The data were subjected to the analysis of variance and Duncan's test $(\mathrm{p} \leq 0.05)$. The results showed significant differences between treatments. The application of irrigation at 75 and $100 \%$ of the requirement allowed to reach the highest plant heights with averages between 53.37 to $57.44 \mathrm{~cm}$ and leaf length with values between 11.89 and $11.37 \mathrm{~cm}$. In addition, these treatments have shown better agronomic behavior, reporting between 2500 to 3500 grains $/ \mathrm{m}^{2}$ and therefore better yields $\left(>120 \mathrm{~g} / \mathrm{m}^{2}\right)$.
\end{abstract}

Keywords: water stress, water deficit, evapotranspiration, yield, Triticum aestivum.

${ }^{1}$ Universidad Nacional Toribio Rodríguez de Mendoza de Amazonas, Instituto de Investigación para el Desarrollo Sustentable de Ceja de Selva, Chachapoyas, Perú

2Universidade Federal de Viçosa, Departamento de Entomologia, Viçosa, Brasil

"Autor de correspondencia. E-mail: juan.neri@untrm.edu.pe 


\section{INTRODUCCIÓN}

El estrés en plantas es generado por factores externos (bióticos y abióticos) que tienen impacto negativo sobre el crecimiento, desarrollo y rendimiento de las plantas, requiriendo que estas desarrollen una serie de mecanismos fisiológicos para poder adaptarse y sobrevivir bajo determinadas condiciones de estrés. El factor de estrés más frecuente es la escasez de agua, el cual reduce el potencial hídrico y la turgencia de la planta, afectando el cierre de estomas, el intercambio de gases, la actividad fotosintética y el movimiento de los nutrientes en la planta (Bosco de Oliveira et al., 2013). El trigo es uno de los tres granos más producidos y consumidos en el mundo junto con el arroz y maíz. Es uno de los cultivos más importantes del mundo y representa aproximadamente el $30 \%$ de la producción total de cereales del mundo cada año (USDA, 2015). Debido a que es un cultivo importante para la alimentación humana, tiene un amplio rango de adaptabilidad en varios ambientes alrededor del mundo (Plana et al., 2006). Sin embargo, las plantas de trigo son sensibles a la sequía durante el período de crecimiento y la etapa más crítica es entre el espigado y llenado de grano (Zadoks et al., 1974).

La escasez de recursos hídricos y los altos costos de riego de los cultivos obligan a los agricultores a cambiar sus métodos de manejo con el fin de obtener producciones más rentables reduciendo las dotaciones hídricas que los cultivos requieren para su adecuado crecimiento y producción. Ante esto, es necesario comprender el impacto de la escasez de agua en el rendimiento y la calidad de los cultivos, y tomar como factor la evapotranspiración de cultivo, para establecer un riego controlado a fin de optimizar el recurso hídrico y reducir la cantidad de agua que se aplica a los cultivos (Sánchez y Torrecillas, 1995; Ramos, 1999). El riego deficitario controlado es una estrategia de aplicación del agua, que permite reducir los aportes hídricos en las fases fenológicas donde el déficit hídrico controlado no afecta sensiblemente a la producción y calidad de la cosecha (Sánchez, 2009).

Para poder estimar los requerimientos de riego, es necesario conocer la evapotranspiración del cultivo (ETc) y la precipitación efectiva (PE) (Ojeda et al., 2007). La evapotranspiración (ET) es la combinación de dos procesos: la evaporación (pérdida del agua en la superficie del suelo) y transpiración de las plantas, siendo una variable primordial en los estudios hidrológicos, modelos de cambio climático y producción de alimentos (Rangel et al., 2013). Además, los efectos combinados de la transpiración del cultivo y la evaporación del suelo se integran en un coeficiente único del cultivo $(\mathrm{Kc})$, siendo indicadores para conocer los requerimientos hídricos de las plantas para la planificación del riego (Allen et al., 2006).

En base a lo expuesto, el presente estudio tuvo por objetivo evaluar el efecto del estrés por déficit hídrico sobre las características morfológicas y agronómicas del cultivo de trigo, bajo condiciones controladas. Además, determinar el régimen de riego que generará resultados similares comparados con el desarrollo del cultivo exento de condiciones de estrés hídrico. Los resultados permitirán establecer una estrategia más razonable sobre el uso del agua y contribuir a la adaptación y mitigación del cambio climático.

\section{MATERIALES Y MÉTODOS}

La presente investigación se desarrolló en las instalaciones de la Universidad Nacional Toribio Rodríguez de Mendoza, ubicado en el distrito de Chachapoyas, Amazonas (Perú). La zona presenta un clima variado con presencia de lluvias en los meses de diciembre a abril, y la temperatura media oscila entre 11 a $\operatorname{los} 23^{\circ} \mathrm{C}$.

\section{Instalación del experimento}

Se preparó sustrato a base de tierra agrícola (textura franca) y humus en la relación 3:1 y se depositaron en 24 macetas de $13 \mathrm{~kg}$ de capacidad (unidades experimentales de $660 \mathrm{~cm}^{2}$ ). Las macetas se colocaron dentro de un microtunel cubierto con plástico UV, considerando un diseño experimental completamente aleatorizado con arreglo factorial, con tres repeticiones y ocho tratamientos (Tabla 1).

Las semillas de trigo fueron sometidas a tratamiento pre-germinativo mediante remojo en agua por un perio- 
Tabla 1. Descripción de tratamientos del experimento

\begin{tabular}{ccc}
\hline \multirow{2}{*}{ Tratamientos } & \multicolumn{2}{c}{ Descripción } \\
\cline { 2 - 3 } & $\begin{array}{c}\text { Régimen de } \\
\text { riego (\%) }\end{array}$ & $\begin{array}{c}\text { Variedad de } \\
\text { trigo }\end{array}$ \\
\hline T1 & 25 & Gavilán \\
T2 & 25 & San Isidro \\
T3 & 50 & Gavilán \\
T4 & 50 & San Isidro \\
T5 & 75 & Gavilán \\
T6 & 75 & San Isidro \\
T7 & 100 & Gavilán \\
T8 & 100 & San Isidro \\
\hline
\end{tabular}

do de 24 horas, luego se efectuó la siembra al voleo en cada unidad experimental. Pasados 30 días se realizó el desahíje o raleo dejando un aproximado de 36 plantas por maceta.

La cantidad de agua necesaria para el riego del trigo en distintas etapas de crecimiento (Tabla 2a) se calculó utilizando la evapotranspiración del cultivo de trigo (ETc) y el requerimiento de riego o lámina bruta de riego (RR) mediante las ecuaciones 1 y 2 (Allen, 2006).

$\mathrm{ETc}=\mathrm{ETo} \times \mathrm{Kc}$
Donde:

$\mathrm{ETc}=$ evapotranspiración del cultivo

Eto $=$ evapotranspiración de referencia (ETo), depende solo del clima de la zona. Para el presente estudio se calculó con datos promediados reportados por la estación experimental de Chachapoyas ubicado en la universidad (periodo 2012-2014), para el caso actual se usó una evapotranspiración de 4,40 mm/día.

$\mathrm{Kc}=$ coeficiente único del cultivo. Los coeficientes utilizados fueron descritos por Garay (2011) para el cultivo de trigo.

$\mathrm{RR}=\mathrm{ETc} /$ Eficiencia de riego $\times 100$

Donde:

$\mathrm{RR}=$ Requerimiento de riego

$\mathrm{ETc}=$ evapotranspiración del cultivo

La eficiencia de riego considerada fue de $90 \%$.

Para el cálculo de los regímenes de riego (cantidad de agua aplicada en cada tratamiento) (Tabla 2b) se usó la equivalencia de $1 \mathrm{~mm}$ que es igual a $11 / \mathrm{m}^{2}$, lo cual se utilizó para aplicar el riego durante todo el ciclo del

Tabla 2. (a) Coeficiente único del cultivo (Kc), evapotranspiración de cultivo (ETc) y requerimiento de agua para riego (RR) en el cultivo de trigo y, (b) régimen de riego según tratamiento

\begin{tabular}{|c|c|c|c|c|c|c|c|c|c|c|}
\hline \multirow[t]{2}{*}{ (a) } & \multirow{2}{*}{$\begin{array}{l}\text { Días después } \\
\text { de la siembra }\end{array}$} & \multirow{2}{*}{ Kc } & \multirow{2}{*}{$\begin{array}{l}\text { ETc } \\
\text { (l) }\end{array}$} & \multirow{2}{*}{$\begin{array}{l}\text { RR } \\
\left(1 / \mathrm{m}^{2}\right)\end{array}$} & \multirow{2}{*}{$\begin{array}{l}\text { RR } \\
\left(\mathrm{l} / \mathbf{0 , 0 6 6} \mathrm{m}^{2}\right)\end{array}$} & \multirow[t]{2}{*}{ (b) } & \multicolumn{4}{|c|}{ Régimen de riego } \\
\hline & & & & & & & $25 \%$ & $50 \%$ & $75 \%$ & $100 \%$ \\
\hline & 10 & 0,250 & 1,100 & 1,222 & 0,081 & & 0,020 & 0,040 & 0,061 & 0,081 \\
\hline & 20 & 0,360 & 1,580 & 1,760 & 0,116 & & 0,029 & 0,058 & 0,087 & 0,116 \\
\hline & 30 & 0,500 & 2,200 & 2,444 & 0,162 & & 0,040 & 0,081 & 0,121 & 0,162 \\
\hline & 40 & 0,650 & 2,860 & 3,178 & 0,210 & & 0,053 & 0,105 & 0,158 & 0,210 \\
\hline & 50 & 0,780 & 3,430 & 3,813 & 0,252 & & 0,063 & 0,126 & 0,189 & 0,252 \\
\hline & 60 & 0,900 & 3,960 & 4,400 & 0,291 & & 0,073 & 0,145 & 0,218 & 0,291 \\
\hline & 70 & 0,980 & 4,310 & 4,791 & 0,317 & & 0,079 & 0,158 & 0,238 & 0,317 \\
\hline & 80 & 1,040 & 4,580 & 5,084 & 0,336 & & 0,084 & 0,168 & 0,252 & 0,336 \\
\hline & 90 & 1,090 & 4,800 & 5,329 & 0,352 & & 0,088 & 0,176 & 0,264 & 0,352 \\
\hline & 100 & 1,110 & 4,880 & 5,427 & 0,359 & & 0,090 & 0,179 & 0,269 & 0,359 \\
\hline & 110 & 1,120 & 4,930 & 5,476 & 0,362 & & 0,090 & 0,181 & 0,271 & 0,362 \\
\hline & 120 & 1,080 & 4,750 & 5,280 & 0,349 & & 0,087 & 0,175 & 0,262 & 0,349 \\
\hline & 130 & 0,980 & 4,310 & 4,791 & 0,317 & & 0,079 & 0,158 & 0,238 & 0,317 \\
\hline
\end{tabular}


cultivo (siembra-cosecha).

Durante el desarrollo del cultivo el riego se aplicó por gravedad mediante un pulverizador. El riego se realizó de forma fraccionada ( 2 a 3 veces por día), considerando la etapa del cultivo y los tratamientos.

\section{Variables evaluadas}

Los datos se recolectaron de 10 plantas centrales de cada unidad experimental, midiendo los siguientes parámetros:

- $\quad$ Altura de planta (AP, cm): Se midió la longitud del tallo desde la superficie del suelo hasta la última espiguilla de la espiga.

- $\quad$ Longitud de hoja y de raíz (L, cm): Se midió la longitud de la hoja y raíz más larga.

- Número de espigas $/ \mathrm{m}^{2}$ : Se determinó al contar el número de total de tallos fértiles (NTF) con espiga y dividido entre el área cosechada neta $\left(0.10 \mathrm{~m}^{2}\right)$; luego se proyectó a $1 \mathrm{~m}^{2}$.

- $\quad$ Número de granos por espiga (G/E): Se determinó al contar y promediar el número de granos por espiga.

- $\quad$ Número de granos $/ \mathrm{m}^{2}\left(\mathrm{G} / \mathrm{m}^{2}\right)$ : Se calculó al multiplicar el número de espigas $\mathrm{m}^{2}$ por el número de granos por espiga $\left(\mathrm{E} / \mathrm{m}^{2} \times \mathrm{G} / \mathrm{E}\right)$.

- $\quad$ Rendimiento $\left(\mathrm{g} / \mathrm{m}^{2}\right)$ : Se obtuvo al determinar el peso del grano después del desgrane de la muestra de la parcela neta $\left(0.10 \mathrm{~m}^{2}\right)$; luego se proyectó a $1 \mathrm{~m}^{2}$.

\section{Análisis de datos}

Se realizó un análisis de varianza y las diferencias entre las medias se evaluó mediante la prueba de Duncan al $5 \%$ de probabilidad de error. Para el procesamiento de los datos se utilizó el programa estadístico InfoStat versión 2018.

\section{RESULTADOS}

\section{Comportamiento morfológico}

El análisis de las variables morfológicas determina la existencia de diferencias significativas $(\mathrm{p} \leq 0,05)$ por la interacción de factores (variedad $\mathrm{x}$ régimen de riego). La tabla 3 muestra que la var. Gavilán con un régimen de riego del $100 \%$ alcanzó la mayor altura promedio con $57,44 \mathrm{~cm}$, mientras que la altura más baja se presentó en los tratamientos con régimen de riego del 25 $\%$, con $27,46 \mathrm{~cm}$ en la var, Gavilán y $26,08 \mathrm{~cm}$ en la var. San Isidro. Las hojas de mayor longitud se registraron en los tratamientos 5 (régimen de riego del 75 $\%$ ), 7 y 8 (régimen de riego del $100 \%$ ) con valores entre 11,37 y $11,89 \mathrm{~cm}$, en cambio la menor longitud se mostró en la var. San Isidro con régimen de riego del $50 \%$, cuyo valor fue de $8,17 \mathrm{~cm}$. La mayor longitud de raíz se registró en la var. Gavilán con régimen de riego del $25 \%(31,64 \mathrm{~cm})$ y $50 \%(32,45 \mathrm{~cm})$, en tanto el menor promedio se presentó en el tratamiento 6 (régi-

Tabla 3. Efecto del régimen de riego sobre variables morfológicas de dos variedades de trigo

\begin{tabular}{cccc}
\hline Tratamientos & Altura de planta & Longitud de hoja & Longitud de raíz \\
\hline T1 & $27,46 \mathrm{~d}$ & $9,22 \mathrm{~cd}$ & $31,64 \mathrm{a}$ \\
T2 & $26,08 \mathrm{~d}$ & $8,77 \mathrm{~cd}$ & $29,13 \mathrm{ab}$ \\
T3 & $36,47 \mathrm{c}$ & $8,84 \mathrm{~cd}$ & $32,45 \mathrm{a}$ \\
T4 & $38,33 \mathrm{c}$ & $8,17 \mathrm{~d}$ & $30,27 \mathrm{ab}$ \\
T5 & $53,42 \mathrm{a}$ & $11,89 \mathrm{a}$ & $30,10 \mathrm{ab}$ \\
T6 & $43,71 \mathrm{~b}$ & $9,41 \mathrm{~b}$ & $27,33 \mathrm{~b}$ \\
T7 & $57,44 \mathrm{a}$ & $11,89 \mathrm{a}$ & $29,64 \mathrm{ab}$ \\
T8 & $53,37 \mathrm{a}$ & $11,37 \mathrm{a}$ & $30,11 \mathrm{ab}$ \\
\hline CV (\%) & 26,92 & 15,53 & 14,65 \\
\hline
\end{tabular}

Medias con letras diferentes en la misma columna indican diferencias significativas (Duncan, $\mathrm{p} \leq 0,05$ )

men de riego del $75 \%$ ) var. San Isidro con $27,33 \mathrm{~cm}$.

\section{Comportamiento agronómico}

El análisis de los datos indica que los factores influyeron significativamente $(\mathrm{p} \leq 0,05)$ sobre las características agronómicas (Tabla 4). El número de espigas por $\mathrm{m}^{2}$ registrado en el tratamiento 7 fue significativamente superior (500 espigas $\left./ \mathrm{m}^{2}\right)$ en comparación con el valor informado en el tratamiento $2\left(383\right.$ espigas $\left./ \mathrm{m}^{2}\right)$. En cuanto al número de granos por espiga, el valor máximo se registró en los tratamientos 7, 8 (régimen

76 Rev. de investig. agroproducción sustentable 5(1): 73-79, 2021 ISSN: 2520-9760 
Tabla 4. Efecto del régimen de riego sobre variables agronómicas de dos variedades de trigo.

\begin{tabular}{ccccc}
\hline Tratamientos & $\begin{array}{c}\text { Número de } \\
\text { espigas/m }\end{array}$ & $\begin{array}{c}\text { Número de granos por } \\
\text { espiga }\end{array}$ & $\begin{array}{c}\text { Número de } \\
\text { granos/m }\end{array}$ & Rendimiento $\left(\mathbf{g} / \mathbf{m}^{\mathbf{2}}\right)$ \\
\hline T1 & $436,67 \mathrm{ab}$ & $0,33 \mathrm{~d}$ & $150,00 \mathrm{~h}$ & $7,21 \mathrm{f}$ \\
T2 & $383,33 \mathrm{~b}$ & $2,67 \mathrm{c}$ & $981,67 \mathrm{~g}$ & $48,65 \mathrm{e}$ \\
T3 & $476,43 \mathrm{ab}$ & $2,75 \mathrm{c}$ & $1359,67 \mathrm{f}$ & $67,20 \mathrm{~d}$ \\
T4 & $463,33 \mathrm{ab}$ & $4,22 \mathrm{bc}$ & $1920,59 \mathrm{e}$ & $96,13 \mathrm{c}$ \\
T5 & $480,00 \mathrm{ab}$ & $6,47 \mathrm{ab}$ & $2588,46 \mathrm{~b}$ & $133,10 \mathrm{~b}$ \\
T6 & $446,67 \mathrm{ab}$ & $4,47 \mathrm{bc}$ & $1971,26 \mathrm{~d}$ & $98,77 \mathrm{c}$ \\
T7 & $500,00 \mathrm{a}$ & $6,87 \mathrm{a}$ & $3428,33 \mathrm{a}$ & $175,29 \mathrm{a}$ \\
T8 & $420,00 \mathrm{ab}$ & $6,55 \mathrm{a}$ & $2533,27 \mathrm{c}$ & $129,60 \mathrm{~b}$ \\
\hline CV $(\%)$ & 12,76 & 22,92 & 0,45 & 2,05 \\
\hline
\end{tabular}

Medias con letras diferentes en la misma columna indican diferencias significativas (Duncan, $\mathrm{p} \leq 0,05$ )

de riego del $100 \%$ ), seguido por el tratamiento 5 (régimen de riego del $75 \%$ ), con un valor promedio entre 6,47 y 6,87 . Además, los tratamientos anteriores han llevado a un registró entre 2500 a 3500 granos $/ \mathrm{m}^{2}$ y consecuentemente a un mayor rendimiento $/ \mathrm{m}^{2}$, con un promedio superior a $120 \mathrm{~g} / \mathrm{m}^{2}$.

\section{DISCUSIÓN}

La evapotranspiración y el requerimiento hídrico se ha estudiado ampliamente para estimar el eficiente uso del agua en el riego, dado que su escasez o poca disponibilidad tienen consecuencias negativas en los cultivos en general. Para ahorrar agua o hacer eficiente su uso, una de las alternativas es el riego deficitario o reducido, estrategia que busca aplicar agua de manera que no se afecte el rendimiento y la calidad de la producción (Serna-Pérez, 2011). Pero para ello, es importante conocer los períodos críticos del cultivo, las condiciones del suelo, el clima, la especie, resistencia, el sistema de riego y otros aspectos de consideración (Zapata y Segura, 2004).

La disponibilidad del recurso hídrico es primordial para el desarrollo de una planta, influyendo tanto en el crecimiento como en el rendimiento. Características como altura de planta y longitud de hoja alcanzaron los valores más altos bajo regímenes de riego del 75 y 100 $\%$. Por su parte, las raíces de mayor longitud se registraron en los tratamientos con regímenes de riego del 25 y $50 \%$. La reducción en el crecimiento foliar es una de las respuestas que las plantas de trigo experimentan frente al estrés hídrico, pero además presentan una reducción en el nivel de fotosíntesis debido a la poca disponibilidad de agua (Potters et al., 2007; Shao et al., 2008). En plantas de trigo var. INCA TH 4 se describió una reducción significativa de su altura y longitud de raíz cuando se regaron al $50 \%$ de su requerimiento hídrico comparado con los niveles que alcanzaron al ser regadas al $100 \%$ de la ETc (Dell'Amico et al., 2016).

Los resultados muestran que la falta de disponibilidad de agua afecta la relación entre el crecimiento de la raíz y la parte aérea de las plantas, pues ante situaciones de déficit hídrico la raíz continúa su desarrollo mientras que la parte aérea (hojas y tallos) deja de crecer. Esta característica, como es el incremento del desarrollo radicular, puede relacionarse a la necesidad de la planta de explorar zonas más profundas del suelo en buscan agua, situación que una planta con alta disponibilidad de agua no experimenta (Potters et al., 2007; Shao et al., 2008).

En cuanto al comportamiento agronómico, los mejores promedios se registraron bajo regímenes de riego entre 50 a $100 \%$ del requerimiento de agua del cultivo. El número de espigas $/ \mathrm{m}^{2}$ varió entre un mínimo de 383,33 y un máximo de 500 espigas. Estos resultados son superiores a los reportado por Morales (2014), quien en el cultivo de triticale informó promedios de 328 y 313 espigas $/ \mathrm{m}^{2}$ bajo regímenes de riego del 100 y $80 \%$ respectivamente. Por otra parte, Quiroz (2010), reportó que el triticale bajo secano obtuvo mayor número de espigas comparado al cultivo bajo riego, con promedios de 422 y 411 espigas $/ \mathrm{m}^{2}$ respectivamente. Las diferencias en los resultados se pueden relacionar a la especie y/o variedad, la influencia de las condiciones climáticas 
de cada zona y la eficiencia del cultivo para aprovechar el agua de riego (Iturrizaga, 2011; Ruiz, 2012; Fernández, 2013). Además, los resultados demuestran que no es necesario aplicar una dosis alta de riego, pero que se debe regular el uso del agua para una mayor eficiencia. Por otro lado, el número de granos por espiga fue menor en los tratamientos con regímenes de riego inferiores al $50 \%$. Este comportamiento pude ser por efecto del estrés por déficit hídrico previo a la antesis, donde la poca disponibilidad de agua provoca una reducción en el número de granos (EstradaCampuzano et al., 2012).Además, un estrés hídrico generado por una sequía, puede reducir significativamente la expansión de área foliar y la materia seca en un cultivo, afectando las etapas de preantesis (inicio de la floración, espigamiento y espiguilla terminal) y postantesis (floración, llenado de grano y madurez fisiológica) notándose efectos severos en el rendimiento (López-Castañeda \& Richards, 1994).

\section{CONCLUSIONES}

Los regímenes de riego del 75 y 100 \% del requerimiento de agua fueron los más óptimos para el cultivo de trigo, debido a que existió un mejor desarrollo expresado en sus características morfológicas y agronómicas. Por el contrario, regímenes de riego menores al $50 \%$ afectan severamente las etapas de floración, espigamiento y llenado de grano reduciendo la cantidad de grano/espiga y el peso de grano.

\section{CONTRIBUCIÓN DE LOS AUTORES}

Todos los autores participaron en la conceptualización, metodología, investigación, redacción del manuscrito inicial, revisión bibliográfica, y en la revisión y aprobación del manuscrito final.

\section{CONFLICTO DE INTERESES}

Los autores declaran no tener conflicto de intereses

\section{REFERENCIAS BIBLIOGRÁFICAS}

Allen, G. R., S. Pereira, D. Raes, y M. Smith. 2006. Evapotranspiración del cultivo: Guías para la determinación de los requerimientos de agua de los cultivos. Roma (Italia); FAO.

Bosco de Oliveira, A., N. L. Mendes, y E. GomesFilho. 2013. "Comparison between the water and salt stress effects on plant growth and development". Responses of Organisms to Water Stress. DOI: 10.5772/54223.

Dell'Amico, J. M., M. M. Roberqui, E. I. Jerez, D. Morales, y R. Plana. 2016. "Respuesta fisiológica del trigo (Triticum aestivum) cultivar INCA TH 4 al déficit hídrico". Cultivos Tropicales 37 (3): 94-102.

Estrada-Campuzano, G., G. A. Slafer, y D. J. Miralles. 2012. "Differences in yield, biomass and their components between triticale and wheat grown under contrasting water and nitrogen environments". Field Crops Res. 128: 167-179.

Fernández, M. A. 2013. "Estrés hídrico: sus efectos sobre el rendimiento de grano y la eficiencia de uso de agua de trigo pan (Triticum aestivum L.), trigo fideos (Triticum aestivum L.) y triticale ( $X$ Triticosecale, Wittmack)". Revista de la Facultad de Agronomía UNLPam 23 (1): 1-17.

Garay, O. B. 2011. Manual de uso consuntivo del agua para los principales cultivos de los Andes Centrales Peruanos. Lima (Perú): Instituto Geofísico del Perú.

Iturrizaga, C. 2011. Comparativo de líneas de trigo harinero primaveral (Triticuma estivum L.) procedentes del CIMMYT en condiciones de la C.C. de Aco-Concepción. Tesis de Grado. Universidad Nacional del Centro del Perú. Huancayo (Perú).

López-Castañeda, C. y R. A. Richards. 1994. "Variation in temperate cereals in rainfed environments. II. Phasic development and growth". Field Crops Research 37 (1): 63-72.

Morales, J. A. 2014. Evaluación de rendimiento de grano y sus componentes en el cultivo de triticale (X Triticosecale Wittmack) bajo diferentes regímenes de riego. Tesis de Grado. Universidad Autónoma Agraria Antonio Narro. 
Coahuila(México).

Ojeda, B. W., L. Hernández, y I. Sánchez. 2007. Manual para Diseño de Zonas de Riego Pequeñas Requerimientos de riego de los cultivos. Jiutepec (México): Instituto Mexicano de Tecnología del Agua.

Plana, R., M. Álvarez, y M. Varela. 2006. “Evaluación de una colección del género Triticum: trigo harinero (Triticum aestivum ssp. aestivum), trigo duro (Triticum turgidum ssp. durum) y triticale (X Triticum secale Wittmack) en las condiciones del occidente de Cuba". Cultivos Tropicales 27 (4): 49-52.

Potters, G., T. P. Pastemak, Y. Guisez, K. J. Palme, y M. A. K. Jansen. 2007. "Stress induced morfogenic responses: growing out of trouble?" Trends Plant Science 12 (3): 99-105.

Quiroz, J. 2010. Respuesta del rendimiento y sus componentes numéricos en trigo, cebada y triticale bajo riego y secano en post-antesis en el sur de Chile. Tesis de Maestría. Universidad Austral de Chile. Valdivia (Chile).

Ramos, G. C. 1999. "Determinación de funciones de producción y comportamiento del cultivo de la cebolla bajo diferentes láminas de riego y dosis de fertilización fosforada en San Juan de Lagunillas, Mérida, Venezuela". Revista Universidad de Zulia 16: 38-51.

Rangel, J. L., K. Mamadou, H. A. Kelso, y F. Magaña. 2013. "Estimación de la demanda hídrica del trigo y sorgo en el Estado de México mediante la recalibración de KT”. Revista Ciencias Técnicas Agropecuarias 22: 72-76

Ruiz, A. 2012. Comparación entre líneas avanzadas de trigo harinero primaveral (Triticum aestivum L.) CIMMYT 30thESWYT, componentes de rendimiento y adaptación ambiental. Tesis de Grado. Universidad Nacional del Centro del Perú. Huancayo (Perú).

Sánchez, B. M. 2009. Curso AECID. Bases para el manejo de aguas en zonas infradotadas. Relación agua-suelo-planta. La Habana (Cuba):
AECID.

Sánchez, B. M., y A. Torrecillas. 1995. Aspectos relacionados con la utilización de estrategias de riego deficitario controlado en cultivos leñosos. Madrid (España): Ediciones MundiPrensa.

Serna-Pérez, A., J. A. Zegbe, y J. Mena-Covarrubias. 2011. "Rendimiento y calidad de chile seco 'Mirasol' cultivado bajo riego parcial de la raíz". Rev. Chapingo S. Hort. 17(1):19-24.

Shao, H.-B., L.-Y. Chu, C. A. Jaleel y C.-X. Zhao. "Water-deficit stress-induced anatomical changes in higher plants". C. R. Biol. 331 (3): 215-255. DOI: 10.1016/j.crvi.2008.01.002

USDA. 2015. Estimaciones mundiales de la oferta y la demanda agrícolas. Washington D.C. (EEUU): USDA.

Zadoks, J. C., T. T. Chang, y C. F. Konzak. 1974. “A decimal code for the growth stages of cereals". Journal Weed Research 14: 415-421.

Zapata, M., y P. Segura. 2004. Riego deficitario controlado, fundamentos y aplicaciones. Madrid (España): Ediciones Mundi-Prensa. 